\title{
IUPAP Young Scientist Prize in Low Temperature Physics 2017 Call for Nominations
}

Nominations are sought for the Young Scientist Prize in Low Temperature Physics.

The prize is awarded by the International Union of Pure and Applied Physics, through Commission C5 (Low Temperature Physics), http://iupap.org/commissions/ c5-low-temperature-physics/

The prize will be awarded at the 28th International Conference on Low Temperature Physics (LT28), to be held in Gothenburg, Sweden, August 9-16, 2017.

The Prize includes a medal, the sum approved by IUPAP, and an invited presentation to be made at LT28.

The nominee is expected to have made original and outstanding contributions to the field of low temperature physics. If the work was performed in collaboration, the leading personal contribution of the nominee to the achievements must be clearly identifiable. Nominees for the prize should, by January 5, 2017, have a maximum of 8 years of research experience following the award of their doctorate.

Nominations should include:

- A letter of support, of not more than 1000 words, evaluating the nominee's achievements and identifying the specific work to be recognized.

- Curriculum vitae of the candidate, identifying all publications.

- A brief biographical sketch of the candidate, not to exceed two pages.

- Suggested text for the award citation.

Self-nominations will not be considered.

These documents should be submitted as a single file in .pdf format. Nominations should be sent as an email attachment to both the IUPAP C5 Commission Chair, J. Saunders (j.saunders@rhul.ac.uk) and the IUPAP C5 Commission Secretary, W. P. Halperin (w-halperin@northwestern.edu).

Deadline for receipt of nominations is January 5, 2017. 